\title{
The stress-related changes in the cerebral blood flow in newborn rats with intracranial hemorrhage: metabolic and endothelial mechanisms
}

\author{
Olga Sindeeva ${ }^{1 *}$, Ekaterina Borisova ${ }^{2}$, Arkady Abdurashitov ${ }^{1}$, Ekaterina Zhinchenko ${ }^{1}$, \\ Artem Gekalyuk ${ }^{1}$, Maria Ulanova ${ }^{1}$, Aly Esmat Sharif ${ }^{1}$, Victoria Razubaevaa ${ }^{1}$, Sergey Serov ${ }^{1}$, \\ Ludmila Yankovskaya ${ }^{3}$, Valery Tuchin ${ }^{1,4}$, Oxana Semyachkina-Glushkovskaya ${ }^{1}$ \\ ${ }^{1}$ National Research Saratov State University, Astrakhanskaya Str. 83, Saratov 410012, Russia \\ ${ }^{2}$ Institute of Electronics, Bulgarian Academy of Sciences, Tsarigradsko Chaussee 72, Sofia 1784, Bulgaria \\ ${ }^{3}$ Grodno State Medical University, Maxima Gorkogo Str. 80, Grodno 230009, Belorus \\ ${ }^{4}$ Laboratory of Biophotonics, Tomsk State University, Tomsk 634050, Russia \\ *e-mail: mouse-oa@rambler.ru
}

\begin{abstract}
Neonatal brain hemorrhages is a major problem of future generation's health due to the high rate of cognitive disability of newborns after vascular catastrophes in the brain. Despite the public health impact of neonatal brain hemorrhages, the mechanisms underlying in these pathological processes remain unknown. Here, using a model of sound-stress-induced brain hemorrhages (per diapedesis, no per rhexis) in newborn rats and optical methods, we found that brain hemorrhages in newborn rats are accompanied by the increase in perfusion of brain tissues, which closely associated with reducing of cerebral oxygenation and increasing of nitric oxide production in both the brain tissues and blood. We assume that nitric oxide contributes the dilation of cerebral vessels during hypoxia and the increasing of cerebral blood flow in newborn rats with brain hemorrhages. Hypoxic-hyperperfusion during stress-related hemorrhages in newborn animals can be an important protective mechanism against anoxia and critical changes in cerebral hemodynamics. (C) 2016 Samara State Aerospace University (SSAU).
\end{abstract}

Keywords: speckle laser contrast imaging, oxygen saturation, stress-related intracranial hemorrhages, hypoxia, nitric oxide.

Paper \#2804 received 2015.12.10; revised manuscript received 2015.12.30; accepted for publication 2015.12.31; published online 2016.02.02.

\section{References}

1. V. J. Rooks, J. P. Eaton, L. Ruess, G. W. Petermann, J. Keck-Wherley, and R. C. Pedersen, "Prevalence and evolution of intracranial hemorrhage in asymptomatic term infants," AJNR Am J Neuroradiol 29(6), 10821089 (2008).

2. T. Takenouchi, E. Kasdorf, and M. Engel, "Changing pattern of perinatal brain injury in term infants in recent years," Pediatr Neurol 46(2), 106-110 (2012).

3. A. J. Brouwer, F. Groenendaal, and C. Koopman, "Intracranial hemorrhage in full-term newborns: a hospitalbased cohort study," Neuroradiol 52(6), 567-576 (2010).

4. S. Siu, N. S. Kwong, and K. T. So, "A 10-year Review of intracranial hemorrhage in term neonates," HK J Peadiatr (new series) 11(2), 140-146 (2006).

5. B. S. Jhawar, A. Ranger, D. A. Steven, and R. F. Del Maestro, “A follow-up study of infants with intracranial hemorrhage at full-term," Can J Neurol Sci 32(3), 332-339 (2005).

6. S. N. Gupta, A. M. Kechli, and U. S. Kanamalla, "Intracranial hemorrhage in term newborns: management and outcomes," Pediatr Neurol. 40(1), 1-12 (2009).

7. E. H. Whitby, P. H. Griffiths, S. Rutter, M. F. Smith, A. Spriqq, P. Ohadike, N. P. Davies, A. S. Rigby, and M. N. Paley, "Frequency and natural history of subdural heamorrhages in babies and relation to obstetric factor," Lancet 363(9412), 846-851 (2004). 
8. C. B. Looney, J. K. Smith, L. H. Merck, H. M. Wolfe, N. C. Chescheir, R. M. Hammer, and J. H. Gilmore, "Intracranial hemorrhage in asymptomatic neonates: prevalence on MR images and relationship to obstetric and neonatal risk factors," Radiology 242(2), 535-541 (2007).

9. S. Maccari, M. Darnaudery, S. Morley-Fletcher, A. R. Zuena, C. Cinque, and O. Van Reeth, "Prenatal stress and longterm consequences: implications of glucocorticoid hormones," Neurosci. Biobehav 27(1-2), 119-127 (2003).

10. C. Mirescu, J. D. Peters, and E. Gould, "Early life experience alters response of adult neurogenesis to stress," Nat. Neurosci 7(8), 841-846 (2004).

11. P. Ballabh, "Intraventricular hemorrhage in premature infants: mechanism of disease," Pediatr Res 67(1), 1-8 (2010).

12. O. Semyachkina-Glushkovskaya, T. Anishchenko, S. Kapralov, R. Novikov, and K. Skvorcov, "Sex differences in cardiovascular control by nitric oxide in normotensive and hypertensive rats at rest and during stress," Health 2(8), 897-905 (2010).

13. T. G. Anishenko, O. V. Semyachkina-Glushkovskaya, and V. A. Berdnikova "Effect of age and sex on renal hypertension and concentration of nitric oxide in the blood of albino rats," Bulletin of Experimental Biology and Medicine 149(1), 1-4 (2010).

14. R. Hlatky, J. C. Goodman, A. B. Valadka, and C. S. Robertson, "Role of Nitric Oxide in Cerebral Blood Flow Abnormalities After Traumatic Brain Injury," Journal of Cerebral Blood Flow \& Metabolism 23(5), 582-588 (2003).

15. P. J. Goadsby, H. Kaube, and H. L. Hoskin, "Nitric oxide synthesis couples cerebral blood flow and metabolism," Brain Res 595(1), 167-170 (1992).

16. C. Iadecola, and F. Zhang, "Permissive and obligatory roles of NO in cerebrovascular responses to hypercapnia and acetylcholine," Am J Physiol 271(4 Pt.2), R990-R1001 (1996).

17. Committee for the Update of the Guide for the Care and Use of Laboratory Animals, Institute for Laboratory Animal Research, Division on Earth and Life Studies, National Research Council of the National Academies, "Guide for the care and use of laboratory animals. 8th edition," Washington, The National Academies Press (2011).

18. O. Semyachkina-Glushkovskaya, A. Pavlov, J. Kurths, E. Borisova, A. Gisbrecht, O. Sindeeva, A. Abdurashitov, A. Shirokov, N. Navolokin, E. Zinchenko, A. Gekalyuk, M. Ulanova, D. Zhu, Q. Luo, and V. Tuchin, "Optical monitoring of stress-related changes in the brain tissues and vessels associated with hemorrhagic stroke in newborn rats," Biomedical Optics Express 6(10), 4088-4097 (2015).

19. O. Semyachkina-Glushkovskaya, V. Lychagov, O. Bibikova, I. Semyachkin-Glushkovskiy, S. Sindeev, M. Kassim, H. Braun, F. Al-Fatle, L. Al Hassani, and V. Tuchin, "The experimental study of stress-related pathological changes in cerebral venous blood flow in newborn rats assessed by DOCT," Journal of Innovative Optical Health Science 6(3), 1-9 (2013).

20. A. Pavlov, O. Semyachkina-Glushkovskaya, Z. Yang, O. Bibikova, O. Pavlova., Q. Huang, D. Zhu, P. Li, V. Tuchin, and Q. Luo "Multiresolution of pathological changes in cerebral venous dynamics in newborn mice with intracranial hemorrhage: adrenorelated vasorelaxation," Physiol. Meas 35(10), 1983-1999 (2014).

21. A. Abdurashitov, V. Lychagov, O. Sindeeva, O. Semyachkina-Glushkovskaya, and V. Tuchin, "Histogram analysis of laser speckle contrast image for cerebral blood flow monitoring," Frontiers of Optoelectronics 8(2), 187-194 (2015).

22. A. K. Dunn, "Laser speckle contrast imaging of cerebral blood flow," Annals of biomedical engineering 40(2), 367-377 (2012).

23. F. Domoki, D. Zölei, O. Oláh, V. Tóth-Szüki, B. Hopp, F. Bari, and T. Smausz, "Evaluation of laser-speckle contrast image analysis techniques in the cortical microcirculation of piglets," Microvascular research 83(3), 311-317 (2012).

24. N. Liu, X. Cui, D. M. Bryant, G. H. Glover, and A. L. Reiss, "Inferring deep-brain activity from cortical activity using functional near-infrared spectroscopy," Biomed. Opt. Express 6(3), 1074-1089 (2015).

25. T. Alderliesten, P. M. Lemmers, J. J. Smarius, R. E. van de Vosse, W. Baerts, and F. van Bel, "Cerebral oxygenation, extraction, and autoregulation in very preterm infants who develop peri-intraventricular hemorrhage," J. Pediatr 162(4), 698-704 (2013).

26. G. A. Taylor, "New concepts in the pathogenesis of germinal matrix intraparenchymal hemorrhage in premature infants," AJNR Am. J. Neuroradiol 18(2), 231-232 (1997).

27. G. A. Taylor, W. H. Trescher, M. V. Johnston, and R. J. Traysman, "Experimental neural injury in the newborn lamb: a comparison of NMDA receptor blockade and nitric oxide synthesis inhibition on lesion size and cerebral hyperemia," Pediatr Res 38(5), 644-651 (1995).

28. G. Hambleton, and J. S. Wigglesworth, "Origin of intraventricular hemorrhage in the preterm infant," Arch Dis Child 51(9), 651-659 (1976). 
29. J. M. Valdueza, F. Doepp, S. J. Schreiber, B. W. van Oosten, K. Schmierer, F. Paul, and M. P. Wattjes, “What went wrong? The flawed concept of cerebrospinal venous insufficiency," J. Cereb. Blood Flow Metab 33(5), 657-668 (2013).

30. A. Michoulas, S. N. Basheer, E. H. Roland, K. Poskitt, S. Miller, and A. Hill, "The role of hypoxia ischemia in term newborns with arterial stroke," Pediatr Neurol 44(4), 254-258 (2011).

31. P. Bodin, and G. Burnstock, "Synergistic effect of acute hypoxia on flow-induced release of ATP from cultured endothelial cells," Experientia 51(3), 256-259 (1995).

32. Y. Tomiyama, J. E. Brian, and M. M. Todd, "Cerebral blood flow during hemodilution and hypoxia in rats: role of ATP-sensitive potassium channels," Stroke 30(9), 1942-1948 (1999).

33. T. Kiliç, and A. Akakin, "Anatomy of cerebral veins and sinuses," Front Neurol Neurosci 23, 4-15 (2008).

34. J. Volpe, "Intracranial hemorrhage: Germinal matrix hemorrhage," in Neurology of the Newborn, 5th edition Philadelphia, PA, Saunders Elsevier (2008). ISBN: 978-1-4160-3995-2.

\section{Introduction}

Neonatal brain hemorrhages $(\mathrm{BH})$ are among the top ten causes of development of significant cognitive disability (42\%-85\% of those who survive) in newborns [1-5]. It was believed that $\mathrm{BH}$ is typical only for pre-term newborns and is uncommon among term neonates because the incidence of $\mathrm{BH}$ in them is difficult to diagnose due to absence of clear symptoms [6]. However, two magnetic resonance imaging studies of asymptomatic term newborns found 8\% (0.2T, 110 neonates) and $26 \%$ (3T, 88 neonates) incidence of $\mathrm{BH}$ after vaginal delivery $[7,8]$. These results show that $\mathrm{BH}$ in term newborns is much more frequent that earlier assumed.

The reasons and mechanisms underlying $\mathrm{BH}$ are difficult to determine. Potential risk factors for $\mathrm{BH}$ are still poorly studied. Some studies report an association between assisted deliveries and $\mathrm{BH}$ in neonates, but those results are not consistent [1, 7, 8]. Such risk factors as hypertension, smoking, diabetes, atherosclerosis are not suitable for neonates. It is assumed that stress as the main process of adaptation to the environment is a crucial risk factor for $\mathrm{BH}$ in neonates, but the mechanism of stress-related $\mathrm{BH}$ is unclear [9, 10].

In this experimental study on three-day-old rat pups, we aimed to determine the mechanisms underlying stress-induced $\mathrm{BH}$. With this aim, we focused on different levels of the cascade of stress responses: systemic (monitoring of cerebral blood flow - CBF), metabolic (assessment of oxygen saturation of the brain tissues) and molecular (evaluation of nitric oxide content in the brain and blood). Our choice of experimental tasks is caused by main actual questions related to the stress-induced cerebrovascular catastrophes. Many studies showed that abnormalities of the $\mathrm{CBF}$, including fluctuations in the $\mathrm{CBF}$, provide a considerable contribution to $\mathrm{BH}$ in newborns [11]. Nitric oxide is a main factor of vascular stress-reactivity and resistance to traumatic stress [12-14]. In the central nervous system, NO participates in the regulation of $\mathrm{CBF}$ and in the cerebrovascular responses to metabolic activity and to hypercapnia $[15,16]$. But, the exact role of these processes in neonatal $\mathrm{BH}$, especially, in aspect of stress accompanying the birth is not established yet and needs to be thoroughly studied.

\section{Materials and Methods}

Experiments were performed out in newborn mongrel rats 3 days old. All procedures were performed in accordance with the "Guide for the Care and Use of Laboratory Animals" [17]. The experimental protocol was approved by the Committee for the Care and Use of Laboratory Animals at Saratov State University (Protocol H-147, 17.04.2001).

To induce hemorrhagic stroke, the following protocol of sound stress's impact was used (120 dB, 17 $\mathrm{Hz}$ ): $10 \mathrm{sec}-$ sound, then $60 \mathrm{sec}-$ pause. This cycle repeated during $2 \mathrm{~h}$ [18-20].

For measurement of cerebral circulation, the anesthetized rats (isoflurane - inhalant anesthetic) with fixed head and scalp incision (the dura was left intact) were immobilized. Anesthetic depth was assessed by periodically monitoring the rear foot reflex.

To assess the stress-related changes in cerebral hemodynamics, we studied the changes in relative cerebral blood flow (rCBF or perfusion) using a homemade system for laser speckle contrast imaging (LSCI). The raw speckle images were recorded under the following conditions: light source - HeNe laser with the wavelength $632.8 \mathrm{~nm}$; image sensor - CMOS camera Basler acA2500-14gm; imaging lens - Computer M16140-MP2 $16 \mathrm{~mm}$ at F-number equal to 6 , that corresponds to speckle/pixel size ratio of around 2 ; exposure time - $20 \mathrm{~ms}$. The speckle images were recorded for $3 \mathrm{~min}$ at an average frame rate of 40 frames per second. Spatial speckle contrast was calculated as, $K=\sigma /\langle I\rangle$, where $\sigma$ is the standard deviation of intensity fluctuations and $\langle I\rangle$ is the mean intensity within a $5 \times 5$ sliding window. 50 consecutive frames were averaged into one speckle contrast image.

The measurement of cerebral circulation was performed through the fontanel of newborn rats with focus on:

1) the superior sagittal sinus, which is one of four major sinuses collecting blood from the small veins of the brain;

2) the small, optically unresolvable vessels surrounding the sagittal sinus. Information about macro- 
(the sagittal sinus) and microcirculation (capillaries, arterioles, venules) was extracted simultaneously.

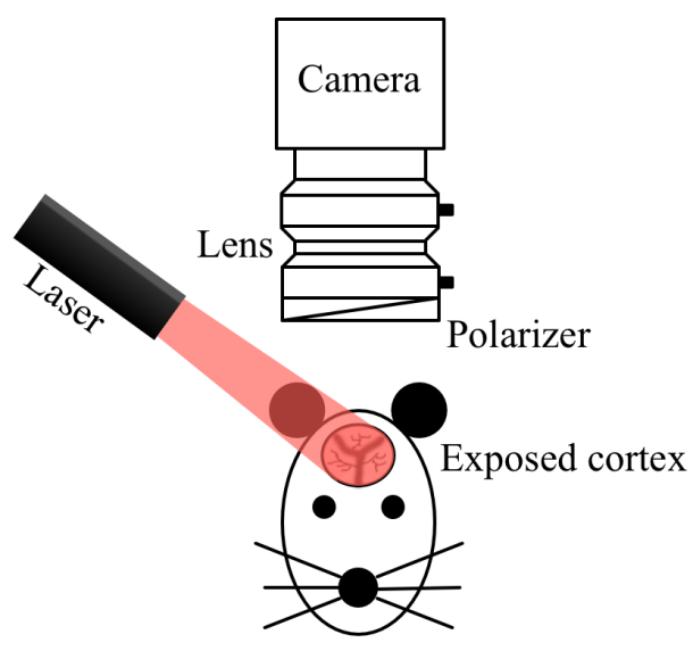

Fig. 1 LSCI system.

Acquisition information from resulting images about flow indexes and vessel's differentiation was performed by histogram analysis of ROI (region of interest). Detailed information about this algorithm and calculation of speckle contrast from raw images itself was explained in our early published article [21] but briefly explanation is following: it was shown several times $[22,23]$ that conventional LSCI system can sense integral erythrocytes motions even in optically unresolvable vessels. Therefore, if we overlay large enough ROI on speckle image (ROI should contain vessels of different caliber and even unresolvable ones) in resulting histogram we can observe clear peaks. Those peaks are related to different vessels with various diameters. By analysis of this histogram and matching each peaks to a specific collection of vessels with particular diameter we can differentiate venous and microcirculatory components in real time during experiments. In this study rCBF was set to reciprocal of speckle contrast.

The changes in oxygen saturation are often associated with the brain injures and are an important for evaluation of severity of these injures [24]. To measure oxygen saturation level $\left(\mathrm{SpO}_{2}\right)$ in the brain tissue we used pulse oximeter model CMS60D (Contec Medical Systems Co., Ltd., Qinhuangdao, China). The system is characterized with a small volume, easy-touse operation and portability, which make it very convenient for the detection of blood hemoglobin oxygen saturation levels of small laboratory animals. Optical sensor is based on dual wavelengths pulse oximetry approach, using $660 \mathrm{~nm}$ and $880 \mathrm{~nm}$ for the $\mathrm{SpO}_{2}$ detection. Display screen of the system could directly show the measured values of the pulse oxygen saturation and pulse rate. Using USB standard for data transmission the measurements could be also visualized on a personal computer using software $\mathrm{SpO}_{2}$ Assistant version 2.7.0. (Contec Medical Systems Co., Ltd.,
Qinhuangdao, China). The software allows as well to store the data in ASCII format for a further analysis.

The content of NO (in vitro) in serum and cerebral tissues (cerebral cortex, cerebellum, hippocampus, brain axis) was determined by the presence of nitrite. The rats were decapitated, the blood samples were collected, the brain was carefully removed and homogenized. Staining was performed using the Griess reagent-Ilosvaya. The color intensity is measured with a spectrophotometer (SF-2000 Bio, Saint Petersburg, Russia, 2002) at fixed wavelength of $538 \mathrm{~nm}$.

\subsection{The protocol of experiments}

The basal values of $\mathrm{SpO}_{2}$ and $\mathrm{rCBF}$ were performed in each healthy newborn rats $(n=24)$. Afterward, each rat underwent to sound stress during $2 \mathrm{~h}$. Next day after stress when brain hemorrhages developed, we performed the recording of $\mathrm{rCBF}$ and $\mathrm{SpO}_{2}$ in the same newborn rats $(n=24)$. Thus, animals were divided into two groups:

1) intact, unstressed newborn rats (the control group);

2) stressed rats $24 \mathrm{~h}$ after stress-off.

The measurement of NO content in the brain tissues and in blood was carried out on other newborn rats: the control group including healthy newborn rats $(n=24)$ and the stressed group $(\mathrm{n}=24)$ including newborn rats $24 \mathrm{~h}$ after stress with brain hemorrhages.

The results were reported as mean \pm standard error of the mean (SEM). Differences from the initial level in the same group were evaluated by the Wilcoxon test. Intergroup differences were evaluated using the MannWhitney test and ANOVA-2 (post hoc analysis with the Duncan's rank test). Significance levels were set at $\mathrm{p}<0.05$ for all analyses.

\section{Results}

\subsection{Stress-induced changes in the cerebral blood flow in newborn rats with brain hemorrhages}

At the first step of our work, we analyzed the stressinduced changes in perfusion and capacitive sectors of cerebral circulation associated with $\mathrm{BH}$ in newborn rats using LSCI system.

The sound stress induced the development of small $\mathrm{BH}$ (average size $0.005 \mathrm{~mm}^{2}$ ) in the cortex and subcortical tissues per diapedesis (no per rhexis), i.e. separated red blood cells squeezed out of the functionally damaged venous vessel walls. Figure 2 (in the right) demonstrates a typical example of diapedesis $\mathrm{BH}$ in the subcortical tissues in newborn rat. All rats survived after sound stress, there were no dead newborn rats. Note, that human newborns with $\mathrm{BH}$ demonstrate 


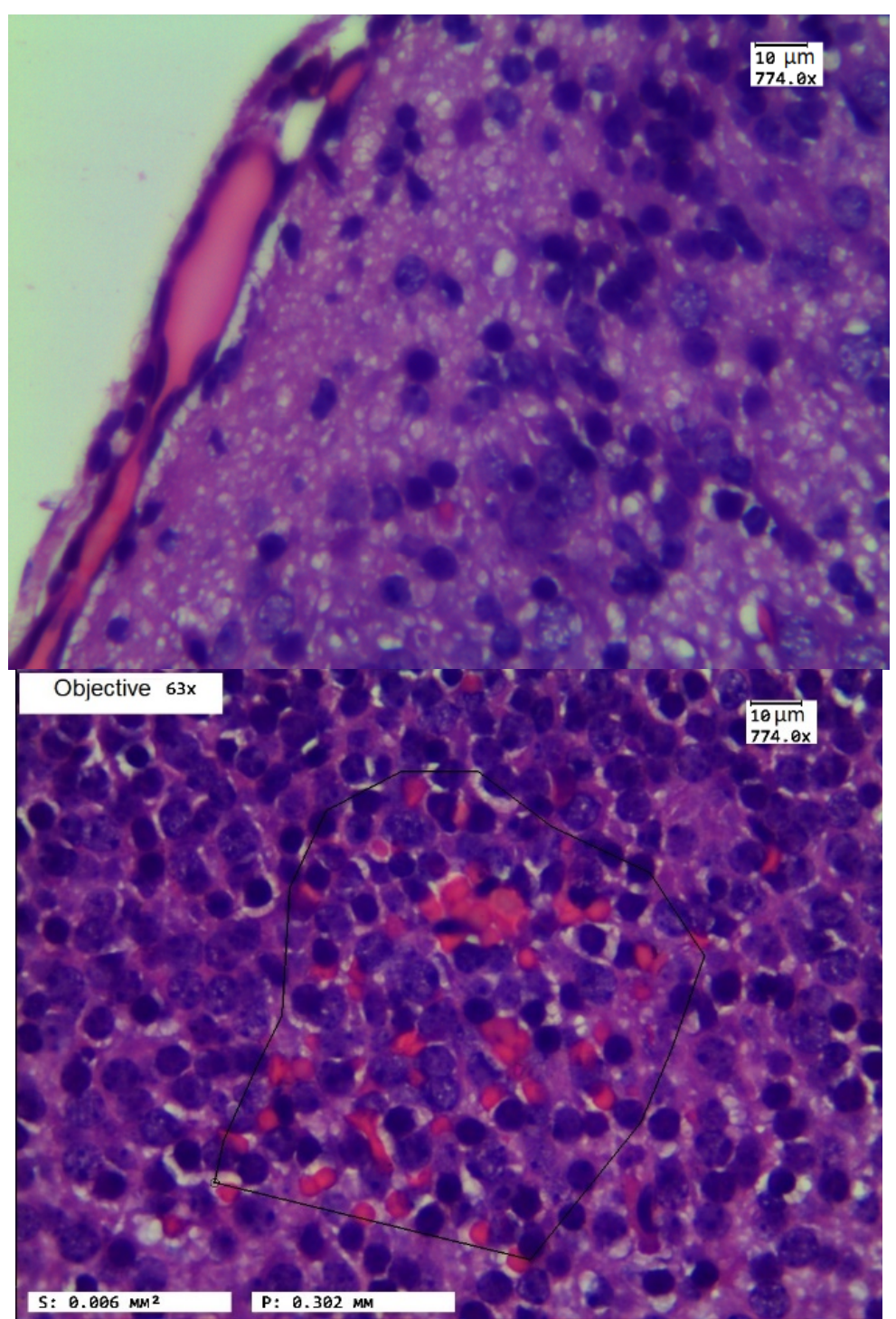

Fig. 2 The typical example of diapedesis (no per rhexis) brain hemorrhage (in the right) in newborn rat (the subcortical area). The normal subcortical tissue of newborn rat from the control group is shown in the left. Hematoxylin \& Eosin staining. Bars represent $10 \mu \mathrm{m}(774.0 \mathrm{X})$.

high survives after such brain injures but with long lasting cognitive disorders [1-5]. The one of possible explanation of this fact is the location of brain hemorrhages in neonates who have usually vascular catastrophes in the cortex, i.e. not in the deep important brain centers controlling vital functions such as heartbeats, breathing and etc. $[3,6]$. Therefore, the consequences of $\mathrm{BH}$ in the intellectual zone of the brain might not be recognized for many years [1-5].

LSCI results showed that newborn rats with $\mathrm{BH}$ vs. healthy animals demonstrated an increased $\mathrm{rCBF}$ in both level of venous and microcirculatory components of cerebral circulation. But, the changes in the sagittal sinus was more pronounced than those in microvessels (Fig. 3). Indeed, after stress exposure rCBF increased in newborn rats by $53 \pm 3 \%(\mathrm{p}<0.05)$ in the sagittal sinus and by $29 \pm 4 \%(p<0.05)$ in microvessels. In our previous study we also obtained that stress-reactivity of cerebral veins are greater than vessels of microcirculatory bed [18].

\subsection{Stress-induced changes in blood oxygen saturation of the brain tissues in newborn rats with brain hemorrhages}

At the second step of this investigation, we studied the metabolic component of cerebral circulation using the assessment of $\mathrm{SpO}_{2}$ of the brain tissues. The results of this series of experiments showed that the stress-related changes in the $\mathrm{CBF}$ in newborn rats was associated with reduced of $\mathrm{SpO}_{2}$ in the brain. The following $\mathrm{SpO}_{2}$ levels averaged for all animals were obtained: $98.1 \pm 0.3 \%$ in the control group and $76.5 \pm 3.0 \%(\mathrm{p}<0.05)$ in newborn rats with $\mathrm{BH}$. Thus, the stress-induced pathological changes in the cerebral circulation was associated with reducing of $\mathrm{SpO}_{2}$ by $22 \%(\mathrm{p}<0.05)$ compared with normal state (Fig. 4). 


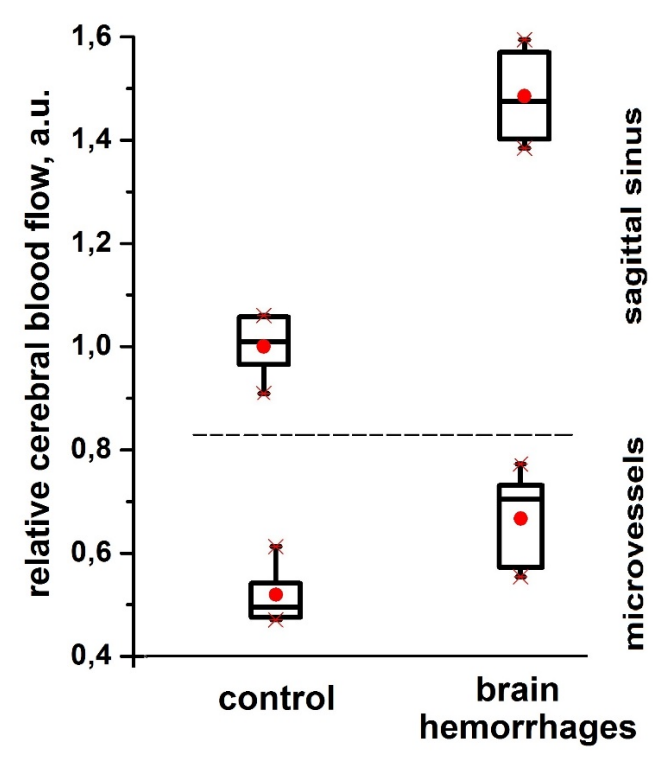

Fig. 3 The relative cerebral blood flow (a.u.) assessed by LSCI in the sagittal sinus and in the microvessels in healthy newborn rats (the control group) and in newborn rat with brain hemorrhages.

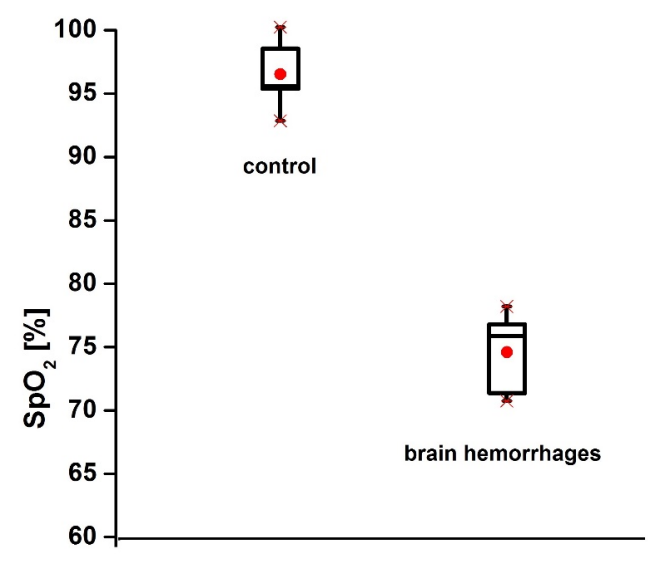

Fig. 4 The $\mathrm{SpO}_{2}(\%)$ in the brain tissues in healthy newborn rats (control group) and newborn rat with brain hemorrhages.

\subsection{Stress-induced changes in NO production in the blood and brain tissue in newborn rats with brain hemorrhages}

At the third step of this work, we analyzed endothelial component of cerebral circulation using spectrophotometric assessment of NO production in the blood and brain tissues in healthy newborn rats (the control group) and in newborn animals with $\mathrm{BH}$.

The results demonstrated that the increased $\mathrm{rCBF}$ and hypoxia, which we obtained in newborn rats with $\mathrm{BH}$ in the first and second parts of this work, were associated with the increase in NO content in all investigated zoned of the brain as well as in the blood (Table 1). So, NO level in newborn rats with ICH was higher in the cortex, cerebellum, hippocampus, brain axis, and in the blood compared with healthy animals.

Table 1 Stress-induced changes in NO $(\mu \mathrm{g} / \mathrm{ml})$ production in the blood and brain tissues in healthy (the control group) and with $\mathrm{BH}$ newborn rats.

\begin{tabular}{lcc}
\hline & $\begin{array}{c}\text { healthy newborn } \\
\text { rats }\end{array}$ & $\begin{array}{c}\text { newborn rats with } \\
\text { ICH }\end{array}$ \\
\hline cortex & $0.23 \pm 0.02$ & $0.36 \pm 0.03^{*}$ \\
cerebellum & $0.27 \pm 0.03$ & $0.32 \pm 0.02 *$ \\
hippocampus & $0.22 \pm 0.04$ & $0.31 \pm 0.05^{*}$ \\
brain axis & $0.20 \pm 0.03$ & $0.28 \pm 0.02 *$ \\
blood & $0.30 \pm 0.07$ & $0.48 \pm 0.05^{*}$ \\
\hline
\end{tabular}

$\mathrm{p}<0.05$ vs.: * - healthy newborn rats (control group)

\section{Discussion}

Here we studied the stress-induced changes in the brain tissues and vessels associated with $\mathrm{BH}$ in newborn rats using LSCI assessment of perfusion (microvessels) and capacitive (the sagittal sinus as a main cerebral vein) components of cerebral circulation as well as a measure of metabolic $\left(\mathrm{SpO}_{2}\right.$ in the brain tissues) and endothelial (level of NO in the brain tissues and in the blood) factors in the regulation of cerebral blood flow.

Our results show that the stress-induced development of $\mathrm{BH}$ in newborn rats is accompanied by the increase in $\mathrm{rCBF}$ in both level of microvessels and venous zones of cerebral circulation. These results are consistent with clinical data suggesting that hyperperfusion is one of mechanisms responsible for brain hemorrhages [25-27]. However, separate LSCI analysis of venous and microcirculatory components of cerebral circulation shows that the maturation of changes in the sagittal sinus were more pronounced than in microcirculatory network $(53 \pm 3 \%$ vs. $29 \pm 4 \%$, $\mathrm{p}<0.05)$. This fact is according with our previous histological data, which showed that the venous vessels are more sensitive to harmful effect of stress than microvessels [18]. In clinical studies also have been shown that neonatal stroke is primary venous infarction due to a weakness of the wall of cerebral veins in neonates [28].

We assume that the increase in $\mathrm{rCBF}$ in the area of microvessels is the consequence of congestion of blood in the cerebral veins and the decreasing of blood outflow from the brain. In our previous histological data [18-20] we showed that the brain hemorrhages in newborn rats are associated with the increase in size of cerebral veins due to accumulation of extensive blood in them. These changes are associated with formation of perivascular edema, i.e. fluid pathway from the vessels. The relaxation of cerebral veins with perivascular edema are markers of accumulation of extensive blood in venous system and suppression of blood outflow from the brain leading to venous insufficiency [29].

The hypoxia is one of main reasons for critical venous pathological changes associated with brain hemorrhages in newborns [30]. Our results suggest that stress-induced increase in $\mathrm{rCBF}$ is accompanied by 
reduction of $\mathrm{SpO}_{2}$ in the brain tissues. Notice, that the hypoxic-hyperperfusion is described also in other studies [31, 32]. A normal physiological response to reduction of oxygen delivery is relaxation of cerebral vessels that activates metabolism in the brain tissues via the increasing of CBF. The particularities of cerebral veins are no muscles and valves in their thin walls, therefore, they have low resistance to critical stretching occurring during blood accumulation in them [33]. The immature brain vessels of newborns have limitation in vasorelaxation capabilities (increase of vessel size). The hypoxia-induced vasorelaxation of cerebral veins causes increasing of cerebral venous pressure [34]. This high pressure can induce easily the rupture of thin walls of immature cerebral veins of newborns [28].

To better understanding of mechanisms underlying relaxation of cerebral vessels during hypoxia in newborn rats with $\mathrm{BH}$, we studied the role of $\mathrm{NO}$ in these processes. Our choice of this vascular factor is caused by the fact that NO is a vasodilator, in the central nervous system, NO participates in the regulation of $\mathrm{CBF}$ and in the cerebrovascular responses to metabolic activity and to hypercapnia $[15,16]$. Thus, NO is complex endothelial factor, which is involved in many levels of regulation of cerebral hemodynamics as well as NO is a key endothelial factor for regulation of vascular tone during stress [12-14].

We show that the stress-induced hypoxichyperperfusion in the brain tissues in newborn rats with $\mathrm{BH}$ is associated with increasing of NO level in the blood and cerebral tissues. The increase NO concentration was the same in different zones of the brain. But, the serum NO level was higher than in the brain that it is possible to explain by the stress-induced increase in NO synthesis in the vessels of all inner organs. In our previous results, we found that the stressinduced increase in NO serum level is a protective mechanism against the significant pressure effect of stress $[12,13]$. Others also demonstrate that the increase of production of major endothelial relaxant such as $\mathrm{NO}$ is one of protective mechanism responsible for increasing of tissue perfusion during hypoxia [32]. Taking into account these data, we believe that the elevated level of NO in the brain and blood is the vascular protective response to hypoxia as mechanism of compensation of oxygen deficiency via the increase in perfusion of brain tissues.

\section{Conclusion}

In summary, our results suggest that stress-induced $\mathrm{ICH}$ in newborn rats is accompanied by the increase in perfusion of brain tissues, which closely associated with reducing of $\mathrm{SpO}_{2}$ in the brain tissues and increasing of NO production in both the brain tissues and blood. We assume that $\mathrm{NO}$ as a major endothelial vasorelaxant may contribute dilation of cerebral vessels under hypoxia that explains the increase in $\mathrm{rCBF}$ in newborn rats with $\mathrm{ICH}$. Thus, hypoxic-hyperperfusion during stress-related hemorrhages in newborn animals can be an important protective mechanism against anoxia and critical changes in cerebral hemodynamics.

\section{Acknowledgments}

This work is supported by Grant of Russian Science Foundation № 14-15-00128. 